\title{
Position-only synthesis of uniformly excited elliptical antenna arrays with minimum element spacing constraint
}

\author{
Hua Guo* D, Guangrui Jing, Mian Dong, Lijian Zhang and Xiaodan Zhang
}

\begin{abstract}
Pattern synthesis of non-uniform elliptical antenna arrays is presented in this paper. Only the element positions of the antenna arrays are optimized by the combination of differential evolution (DE) and invasive weed optimization (IWO) to reduce the peak side lobe level (PSLL) of the radiation pattern. In order to avoid the overlap of the array elements, the minimum spacing of the adjacent elements is constrained. Also, the beam width of the radiation pattern can be constrained effectively. Three elliptical antenna arrays that have 8, 12, and 20 elements are investigated. The synthesis results show that the introduced method can present a good side lobe reduction for the radiation pattern. Compared with other optimization methods, the method proposed in this paper can obtain better performance.
\end{abstract}

Keywords: Pattern synthesis, Elliptical antenna arrays, Position only, Invasive weed optimization

\section{Introduction}

Designs of antennas have been paid more attention in the last few years [1-22]. Also, pattern synthesis of antenna arrays has become a traditional problem [6-20]. Moreover, much attention has been paid to the position-only synthesis of uniformly excited antenna arrays [6-14]. There are two kinds of uniformly excited antenna arrays. One is thinned array [6-10], which is to delete some array elements from a uniform array. These kinds of arrays can easily constrain the spacing of the adjacent elements. The other one is sparse antenna array which means that the array elements are randomly distributed along the antenna array [11-14]. Compared with thinned antenna arrays, sparse antenna arrays can obtain better simulation results. However, in the design of sparse antenna arrays, it is hard to constrain the size of the array aperture, number of the array elements, and the minimum spacing of adjacent elements at the same time.

In recent years, as the development of modern military and defense application, circular and elliptical antenna arrays have become more popular in wireless communication [1520]. Compared with linear antenna arrays, the radiation

\footnotetext{
*Correspondence: xdguohua@163.com

School of Electronics and Information, Xi'an Polytechnic University, Xi'an 710048 , China
}

patterns of the circular and elliptical antenna arrays can cover the entire space. In the design of circular and elliptical antenna arrays, synthesis of non-uniform arrays has been paid more attention [15-20]. In [15], genetic algorithms are proposed to determine optimum weights and element separations that provided a radiation pattern with peak side lobe level reduction with a fixed beam width. As is introduced in [16], Hierarchical Dynamic Local Neighborhood Based PSO (HDLPSO) algorithm is introduced into the design of nonuniform circular antenna arrays. Invasive weed optimization (IWO) is presented in [17] for the design of non-uniform, planar, and circular antenna arrays that can achieve minimum side lobe levels for a specific first null beam width. Biogeography-based optimization (BBO) is presented in [18] for the optimization of non-uniform circular antenna arrays, and the optimization results show that the synthesis of nonuniform circular antenna arrays using the $\mathrm{BBO}$ algorithm provides a SLL reduction better than that obtained by using a genetic algorithm, simulated annealing, and particle swarm optimization. In [20], synthesis of elliptical antenna arrays is introduced by using three different optimization algorithms (self-adaptive differential evolution method, biogeographybased optimization method, and firefly algorithm).

In order to avoid the overlap of the array elements, the space of the adjacent elements must be constrained in 
the design of elliptical antenna arrays. So, the purpose of this paper is to reduce the peak side lobe level of the radiation pattern by optimizing the positions of array elements that fulfil the minimum space constraint. The element positions are optimized by differential invasive weed optimization (DIWO), and low PSLLs of the radiation patterns are obtained. IWO has been widely used in the design of antennas $[17,21,22]$. Usually, IWO has better optimization results than the other optimization methods in the final error level. The rest of the paper is organized as follows: Method and experiment are introduced in Section 2. In Section 3, the mathematical formulation is given. The used optimization algorithms and the optimization steps are briefly described in Section 4 . In Section 5, numerical results and comparisons are given. Finally, the paper is concluded in Section 6.

\section{Method and experiment}

The study of the paper is to introduce a new design method of non-uniform elliptical antenna arrays. First, the design of antenna arrays is expressed as an optimization problem. The structure of an elliptical antenna array is depicted, and the fitness function is given. Also, the formulations of the element positions that can constrain the minimum element spacing are deduced. Second, a modified invasive weed optimization is introduced. Then, it is used to solve the introduced optimization problems. The experiment is based on numerical simulations. To verify the effectiveness of the proposed method, synthesis results and comparisons are made by taking three different antenna arrays. Comparisons show that the proposed algorithm is more efficient than other algorithms. The analysis shows that the proposed method is useful and effective to solve the design of non-uniform elliptical antenna arrays.

\section{Mathematical formulation}

3.1 Structure and array factor of elliptical antenna array The structure of an elliptical antenna array with $N$ elements placed in the $x-y$ plane is given in Fig. 1 . The original point is at the center of the ellipse. The semi-major axis and semi-minor axis lengths are depicted by $a$ and $b$, respectively. The eccentricity of the ellipse is $e$ which is calculated by

$$
e=\sqrt{1-\frac{b^{2}}{a^{2}}}
$$

The array factor of this elliptical antenna array is given by

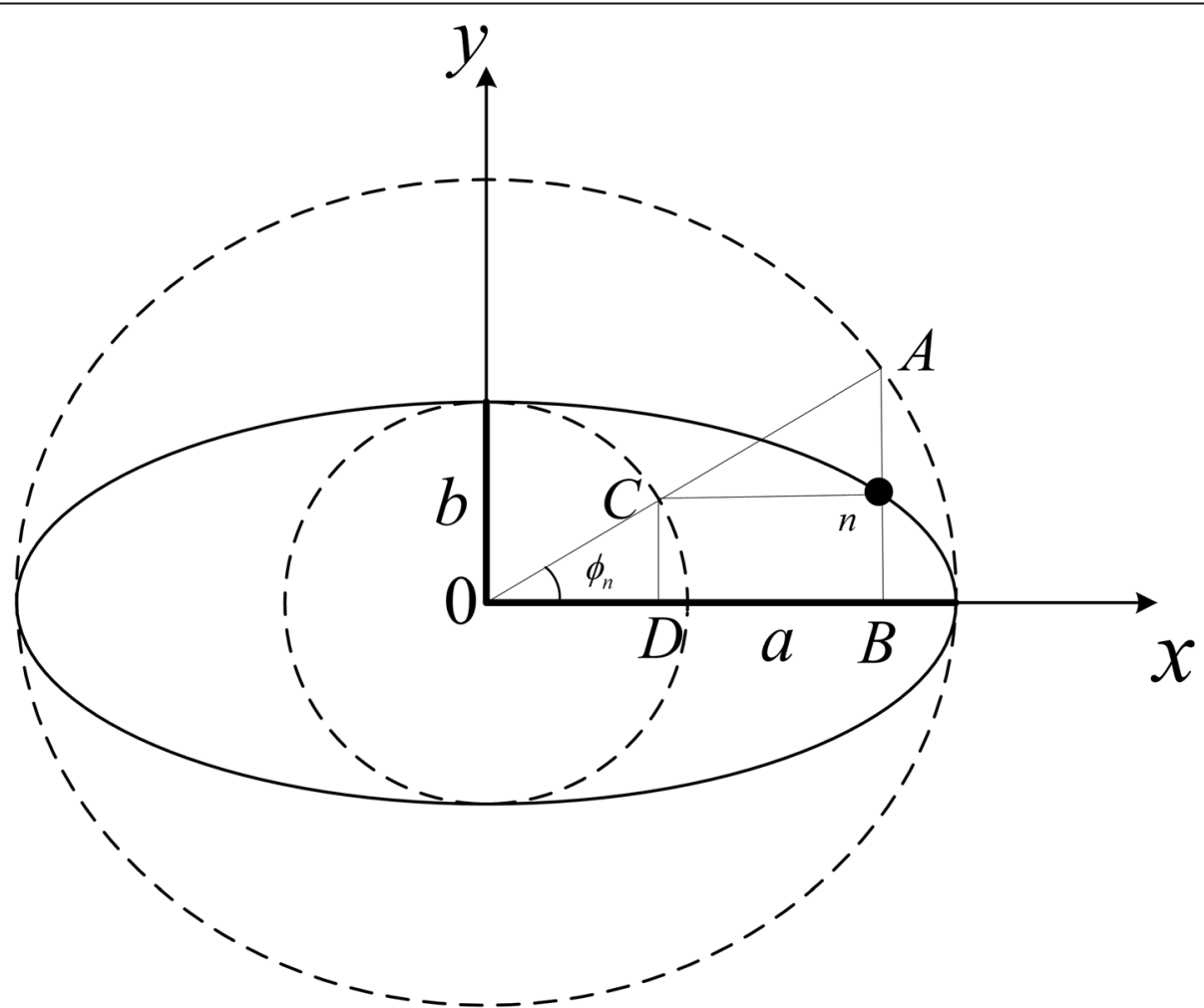

Fig. 1 Structure of elliptical antenna array 
$A F(\theta, \phi)=\sum_{n=1}^{N} I_{n} \exp \left[j\left(k \sin \theta\left(a \cos \phi_{n} \cos \phi+b \sin \phi_{n} \sin \phi\right)\right)+j \alpha_{n}\right]$

where $k=2 \pi / \lambda$ is the wave number. $I_{n}$ and $\alpha_{n}$ represent the excitation amplitude and phase of the $n$th element. $\phi_{n}$ is the angular position of the $n$th element in the $x-y$ plane. $\phi$ is the azimuth angle which is measured from the positive $x$-axis. $\theta$ is the elevation angle measured from the positive $z$-axis. In the following design, the array factor in the $x-y$ plane, i.e., $\theta=90^{\circ}$, is considered. So, the array factor of the elliptical antenna array can be depicted by

$$
A F(\phi)=\sum_{n=1}^{N} I_{n} \exp \left[j\left(k\left(a \cos \phi_{n} \cos \phi+b \sin \phi_{n} \sin \phi\right)\right)+j \alpha_{n}\right]
$$

If the direction of the main beam is $\phi_{0}$, the phase excitation of the $n$th array element can be given by

$$
\alpha_{n}=-k\left(a \cos \phi_{n} \cos \phi_{0}+b \sin \phi_{n} \sin \phi_{0}\right)
$$

\subsection{Fitness function}

In order to obtain radiation patterns with minimum side lobe levels and a specific first null beam width (FNBW), the element positions of elliptical antenna arrays are optimized. The desired function can be given by

$$
E=\left.w_{1} \frac{|A F(\phi)|}{A F_{\max }}\right|_{\phi \in S}+w_{2}\left|\mathrm{FNBW}_{\mathrm{c}}-\mathrm{FNBW}_{\mathrm{d}}\right|
$$

where $w_{1}$ and $w_{2}$ are the weighting factors. $A F_{\max }$ is the maximum value of $|A F(\phi)| . S$ is the side lobe area of the radiation pattern. The calculated first null beam width is defined by $\mathrm{FNBW}_{\mathrm{c}}$, and the desired first null beam width is depicted by $\mathrm{FNBW}_{\mathrm{d}}$.
In order to avoid the overlap of array elements, the minimum spacing of the adjacent elements is depicted by $d_{e}$. So, the objective function can be given by

$$
\left\{\begin{array}{c}
\min \{E\} \\
\text { s.t. } \sqrt{\left(x_{i}-x_{j}\right)^{2}+\left(y_{i}-y_{j}\right)^{2}} \geq d_{e} \\
i, j=1,2, \cdots N ; i \neq j
\end{array}\right.
$$

where $\left(x_{n}, y_{n}\right)$ is the position of the $n$th element which can be calculated by

$$
\left\{\begin{array}{l}
x_{n}=a \cos \phi_{n} \\
y_{n}=b \sin \phi_{n}
\end{array}\right.
$$

In the following procedure, the problem is changed into a maximum problem. So, the fitness function can be defined by

$$
f=\frac{1.0}{1+E}
$$

\subsection{Optimization of element positions}

As is shown in Fig. 1, the length of the elliptical antenna array can be calculated by

$$
L=a \int_{0}^{2 \pi} \sqrt{1-e^{2} \cos ^{2} \phi} d \phi
$$

The length that can allocate array elements is $L-d_{e}$. If array elements are allocated by the minimum spacing constraint $d_{e}$, the length of $(N-1) d_{e}$ is occupied. So, the remaining region over the array aperture to be optimized is given by [11]

$$
S P=L-d_{e}-(N-1) d_{e}=L-N d_{e}
$$

In the determination of element positions, the position of the first element must be determined. The first element should be allocated in the area of $[0, S P]$. As is

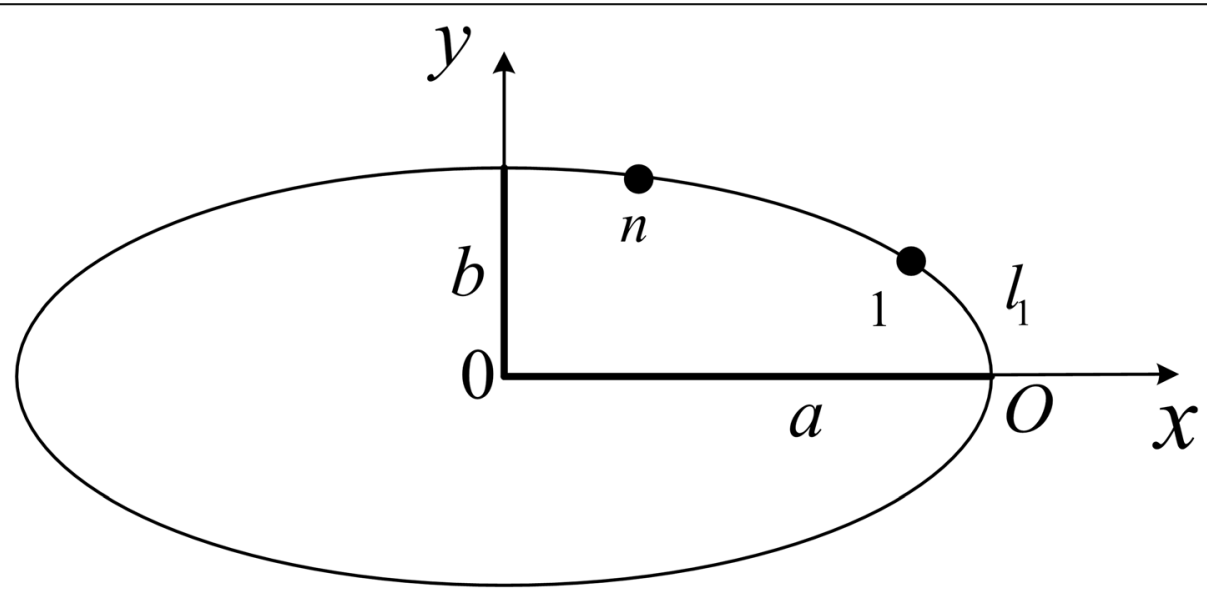

Fig. 2 Elements distribution of elliptical antenna array 
shown in Fig. 2, the length from $O$ to the first element position along the ellipse can be depicted by

$$
l_{1}=S P \times r_{1}
$$

where $r_{1}$ is the random number among the rang of $[0,1]$. Then, $N-1$ real random numbers among the range of $[0$, $S P]$ are calculated by

$c_{i-1}^{\prime}=S P \times r_{i}, i=2,3, \cdots, N(12)$.

where $r_{i}, i=2,3, \cdots, N$ are real random numbers among the range of $[0,1]$. Then, $c_{i}^{\prime}$ are sorted in ascending order and a new vector $\mathbf{C}=\left[c_{1}, c_{2}, \cdots, c_{N-1}\right]$ is obtained, where $c_{1} \leq c_{2} \leq \cdots \leq c_{N-1}$. The length from $O$ to the $n$th element position along the ellipse can be calculated by

$$
\begin{array}{r}
{\left[\begin{array}{c}
l_{2} \\
l_{3} \\
\cdots \\
l_{N}
\end{array}\right]=l_{1}+\left[\begin{array}{c}
c_{1} \\
c_{2} \\
\ldots \\
c_{N-1}
\end{array}\right]+\left[\begin{array}{c}
d_{e} \\
2 d_{e} \\
\ldots \\
(N-1) d_{e}
\end{array}\right]} \\
=l_{1}+\left[\begin{array}{c}
c_{1}+d_{e} \\
c_{2}+2 d_{e} \\
\cdots \\
c_{N-1}+(N-1) d_{e}
\end{array}\right]
\end{array}
$$

If $l_{n}>L, l_{n}$ is updated by

$$
l_{n}=l_{n}-L, n=2,3, \cdots, N
$$

Then, $l_{n}$ is sorted in ascending order and the ultimate $l_{n}$ is obtained. It can be proved that the element spacing between $n$th element and $(n+1)$ th element is $d_{e}+\left(c_{n+1}\right.$ $\left.-c_{n}\right), n=1,2, \cdots, N-1$ which satisfies the minimum spacing constraint of (6).

After $l_{n}, n=1,2, \cdots, N$ are calculated, the angular position $\phi_{n}$ of the $n$th array element can be calculated by

$$
\left\{\begin{array}{c}
l_{n}-a \int_{0}^{\phi_{n}} \sqrt{1-e^{2} \cos ^{2} \phi} d \phi=0 \\
n=1,2, \cdots, N
\end{array}\right.
$$

\section{Optimization algorithm}

IWO and DE are intelligent algorithms which have been used in various kinds of optimization problems [21-24]. In order to improve the synthesis results, invasive weed optimization and differential evolution algorithm are combined for parallel computation. The IWO and DE has data exchange after $m$ iteration steps. The newly introduced algorithm is called DIWO. The flowchart of this optimization method is given by Fig. 3. The optimization procedure can be expressed as follows:

Step 1. The parameter values of the antenna arrays and DIWO are given. A $N \times P$-dimensional matrix $\mathbf{r}_{-}$I and a $N \times d \_$max-dimensional matrix $\mathbf{r}_{-} D$ are chosen

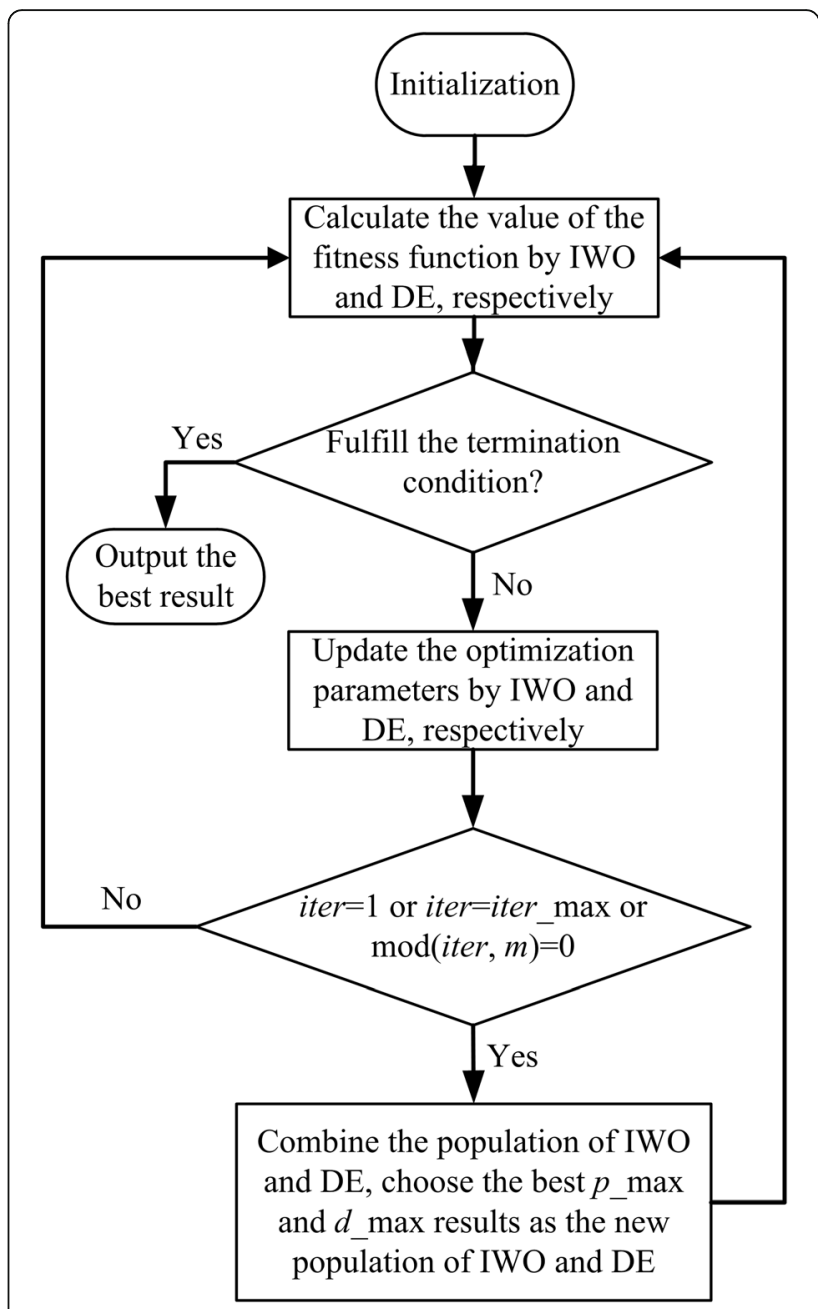

Fig. 3 Flowchart representation of DIWO

as the initial population for IWO and DE, respectively. Let $i$ ter $=1$.

Step 2. The angular position of the array elements is calculated by (15).

Step 3. The radiation patterns of the antenna arrays are calculated by (3). The fitness value is defined by (8) which increases with the decrease of PSLL. The optimized element positions that can produce the best fitness value are preserved as the ultimate result.

Step 4. The optimization parameters $\mathbf{r}_{-}$I and $\mathbf{r}_{-} D$ are updated by IWO and DE.

Step 5. If iter $=1$ or iter $=i$ ter_max or $\bmod ($ iter, $m)=0$, go to step 6; otherwise, go to step 7. Where $\bmod ($ ) is the modulus after division.

Table 1 DIWO parameter values

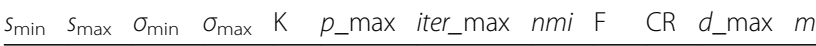
\begin{tabular}{llllllllllll}
\hline 1 & 12 & $10^{-4}$ & 0.1 & 10 & 30 & 300 & 3 & 0.3 & 0.2 & 100 & 5
\end{tabular} 


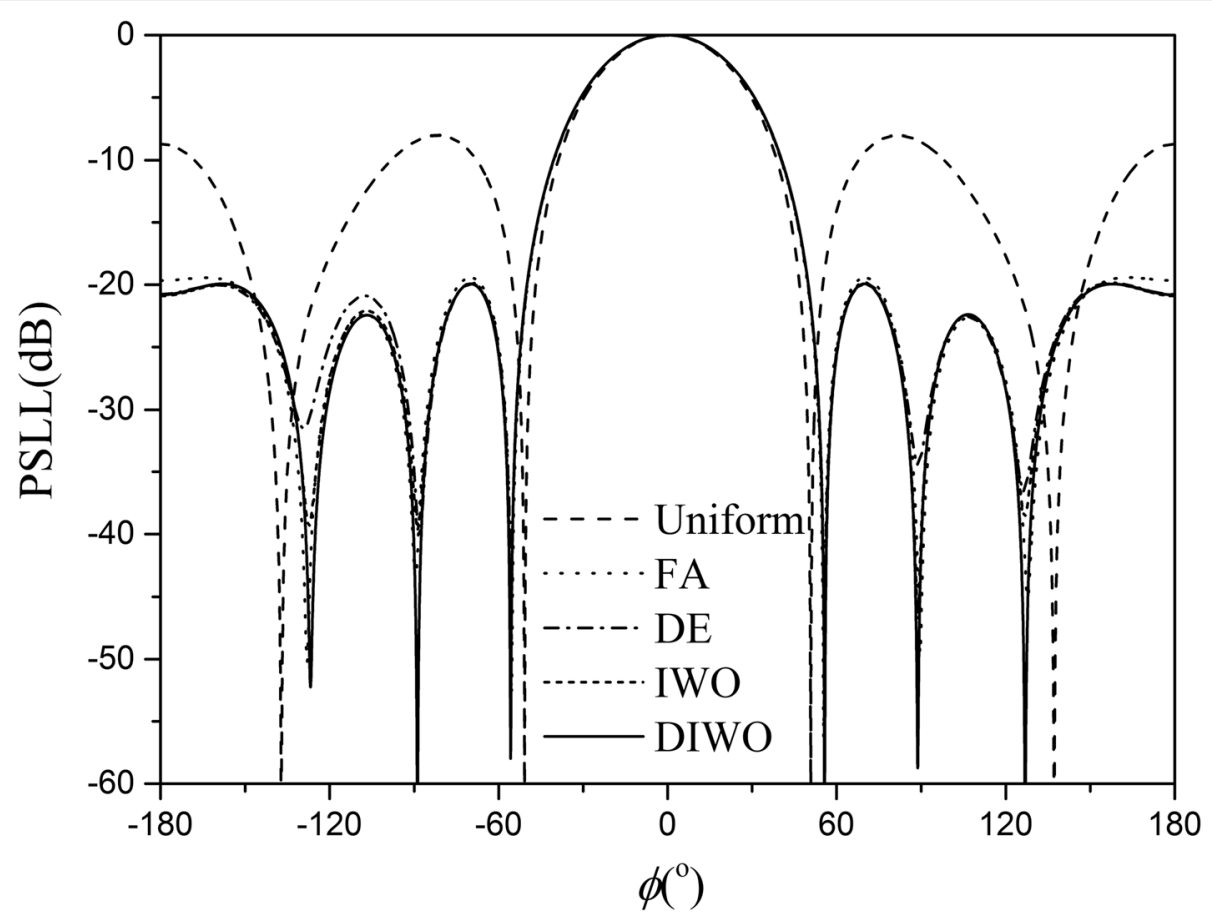

Fig. 4 Radiation pattern of elliptical antenna array with 8 elements

Step 6. Combine all the optimization population of IWO and DE. Choose the best $p \_$max population as the new population of IWO. Choose the best $d \_$max population as the new population of DE.

Step 7. Let iter $=$ iter +1 , if iter $<$ iter_max, go to step 2 ; otherwise, terminate iteration.

\section{Results and discussion}

In this section, several simulation results are given to show the feasibility and effectiveness of the proposed method. The parameters used in DIWO are given in Table 1. The minimum spacing constraint for the adjacent array elements is chosen as $d_{e}=$ $0.15 \lambda$. The direction of the main beam is $\phi_{0}=0^{\circ}$. The weighting factor $w_{1}=1, w_{2}=3$. The positions of the array elements are optimized to get radiation patterns with low side lobe levels. The algorithm is calculated 10 times, and the best result is preserved as the ultimate result.

\subsection{Optimization result of 8-element antenna array}

In the first example, pattern synthesis of elliptical antenna array that has 8 elements is introduced. The semi-major axis length $a$ and eccentricity $e$ of the ellipse are $0.5 \lambda$ and 0.5 , respectively. The desired first null beam width is chosen as $\mathrm{FNBW}_{\mathrm{d}}=111^{\circ}$. The same array is optimized in [20] using self-adaptive differential evolution (SADE) technique, biogeography-based optimization (BBO) and firefly algorithm (FA). The PSLLs of the radiation patterns optimized by these three methods are $-19.12 \mathrm{~dB},-19.40 \mathrm{~dB}$ and $-19.43 \mathrm{~dB}$, respectively. When the elements are uniformly placed on the ellipse, the PSLL of the radiation pattern is $-8.02 \mathrm{~dB}$. Using the method proposed in this paper, the best PSLL of 10 calculation times obtained by DIWO is $-19.91 \mathrm{~dB}$ which is $0.48 \mathrm{~dB}$ lower than that optimized by FA. The beam width of the radiation pattern is $111.5^{\circ}$. The minimum spacing of the adjacent elements is $0.18 \lambda$ which fulfils the minimum spacing constraint of (6). When the same array is optimized by DE and IWO, the best PSLLs are $-19.82 \mathrm{~dB}$ and $-19.89 \mathrm{~dB}$, respectively.

Table 2 Positions of elliptical antenna array with 8 elements

\begin{tabular}{lll}
\hline$N=8$ & $\varphi_{n}(\stackrel{\circ}{)}, n=1,2, \cdots, 8$ & PSLL (dB) \\
\hline Uniform & {$[0,45,90,135,180,225,270,315]$} & -8.02 \\
FA in $[20]$ & {$[30.56,53.93,125.89,149.16,210.99,233.52,306.57,329.13]$} & -19.43 \\
DE & {$[30.95,52.16,127.41,147.68,210.33,234.05,305.69,329.89]$} & -19.82 \\
IWO & {$[30.31,53.45,126.14,149.41,211.36,232.91,307.47,328.85]$} & -19.89 \\
DIWO & {$[30.87,53.11,126.80,149.28,210.84,233.29,306.83,329.04]$} & -19.91 \\
\hline
\end{tabular}




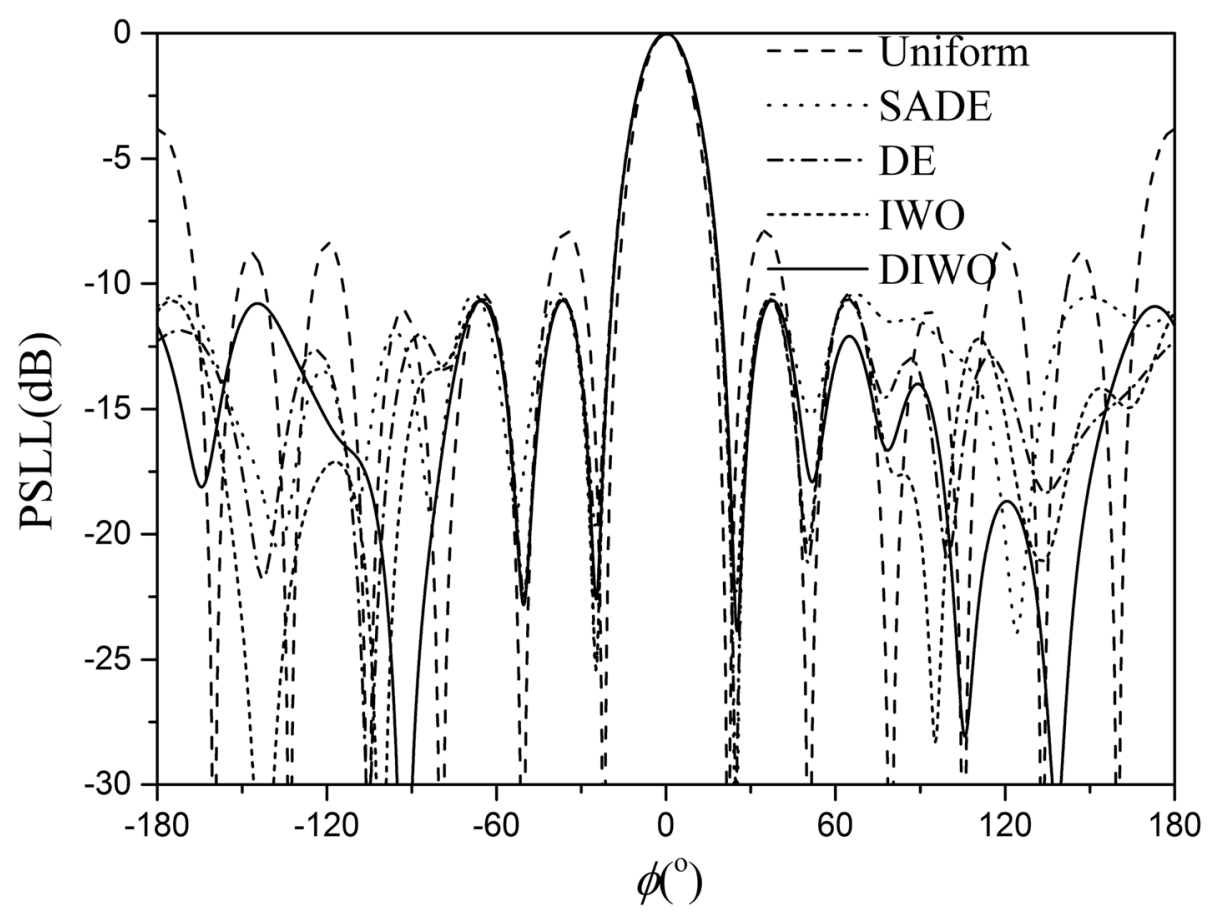

Fig. 5 Radiation pattern of elliptical antenna array with 12 elements

The radiation patterns synthesized by different algorithms are given in Fig. 4. Table 2 shows the element positions of the best synthesis result optimized by different algorithms. The worst PSLL of 10 calculation times optimized by DIWO is $-19.72 \mathrm{~dB}$ while the average PSLL is $-19.81 \mathrm{~dB}$.

\subsection{Optimization result of 12-element antenna array}

In this example, synthesis result of elliptical antenna array that has 12 elements is given. The semi-major axis length and eccentricity of the ellipse are chosen as $1.15 \lambda$ and 0.5 , respectively. The desired first null beam width is $49^{\circ}$. The best PSLL optimized in [20] is $-10.37 \mathrm{~dB}$ by using SADE. The PSLL of the radiation pattern is $-3.82 \mathrm{~dB}$ when the elements are uniformly distributed. Using the method proposed in this paper, the best PSLL obtained by DIWO is $-10.65 \mathrm{~dB}$. The beam width of the radiation pattern is $49.8^{\circ}$. The minimum spacing of the adjacent elements is $0.15 \lambda$. When the same array is optimized by DE and IWO, the best PSLLs are $10.56 \mathrm{~dB}$ and $-10.58 \mathrm{~dB}$, respectively. Figure 5 is the radiation patterns with minimum PSLL synthesized by different algorithms. Table 3 is the element positions of the elliptical antenna array optimized by different algorithms. In this example, the worst PSLL of 10 calculation times obtained by DIWO is $-10.36 \mathrm{~dB}$ and the average PSLL is $-10.56 \mathrm{~dB}$.

\subsection{Optimization result of 20-element antenna array}

In [20], the 20-element elliptical antenna array is optimized by $\mathrm{SADE}, \mathrm{BBO}$, and FA. The semi-major axis length and eccentricity of the ellipse are chosen as $1.6 \lambda$ and 0.5 , respectively. The best PSLL is $-11.27 \mathrm{~dB}$ obtained by FA. In this paper, the same array is optimized by DIWO. The desired beam width of the radiation pattern is $-34^{\circ}$. The best PSLL of the radiation pattern is $-12.21 \mathrm{~dB}$ synthesized by DIWO. The beam width of the radiation pattern is $34.8^{\circ}$. The

Table 3 Positions of elliptical antenna array with 12 elements

\begin{tabular}{|c|c|c|}
\hline$N=12$ & $\varphi_{n} \stackrel{\circ}{)}, n=1,2, \cdots, 12$ & PSLL (dB) \\
\hline Uniform & {$[0,30,60,90,120,180,210,240,270,300,330]$} & -3.82 \\
\hline SADE in [20] & $\begin{array}{l}{[13.57,61.10,101.10,121.03,157.57,180.10,196.06,} \\
203.53,254.08,266.70,327.94,349.25]\end{array}$ & -10.37 \\
\hline DE & $\begin{array}{l}{[14.81,60.24,97.38,139.92,158.98,170.70,197.52,} \\
206.09,227.03,268.34,302.22,341.96]\end{array}$ & -10.56 \\
\hline IWO & $\begin{array}{l}{[14.69,24.14,47.88,90.99,121.35,167.13,197.90,} \\
237.88,274.65,319.45,337.15,346.11]\end{array}$ & -10.58 \\
\hline DIWO & $\begin{array}{l}{[13.32,24.79,42.06,85.26,123.08,167.10,195.78,} \\
242.39,265.25,315.03,338.42,346.93]\end{array}$ & -10.65 \\
\hline
\end{tabular}




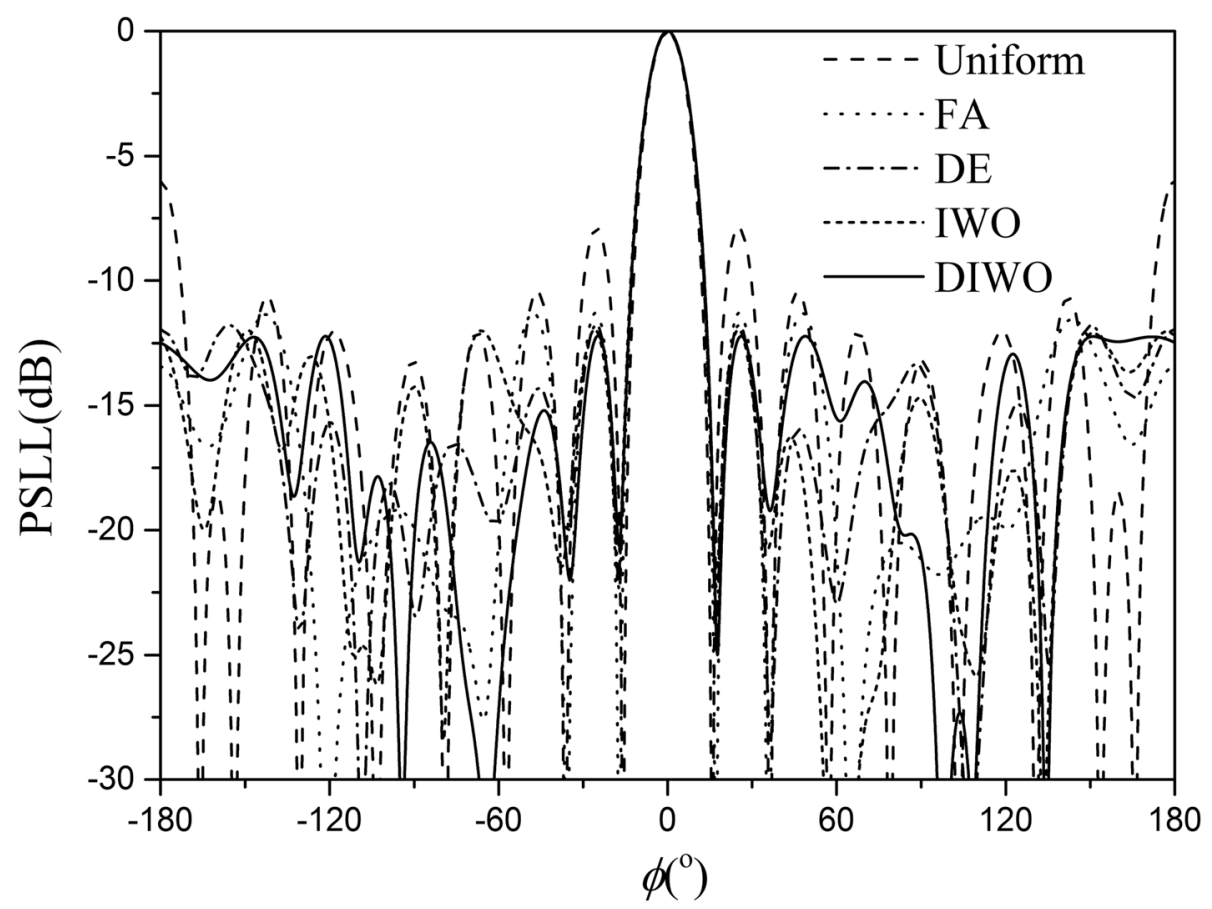

Fig. 6 Radiation pattern of elliptical antenna array with 20 elements

minimum spacing of the adjacent elements is $0.15 \lambda$. When the same array is optimized by DE and IWO, the best PSLLs are $-11.93 \mathrm{~dB}$ and $-11.96 \mathrm{~dB}$, respectively. The radiation patterns synthesized by different algorithms are given in Fig. 6. Table 4 gives the element positions of the elliptical antenna array optimized by different optimization algorithms. In this synthesis example, the worst PSLL of 10 calculation times obtained by DIWO is $-11.77 \mathrm{~dB}$ and the average PSLL is $-11.87 \mathrm{~dB}$.

\section{Conclusions}

Position-only synthesis of uniformly excited elliptical antenna arrays with minimum element spacing constraint has rarely been studied. When the array size and element number are given, it is hard to constrain the minimum spacing of the adjacent elements which is important in the design of antenna arrays. For the first time, pattern synthesis of non-uniform elliptical antenna arrays with minimum spacing constraint is introduced. The comparisons show that the design of non-uniform elliptical antenna arrays using the proposed method presents a good side lobe reduction in the radiation pattern for the optimized problem. Also, according to the real requirement of antenna array design, the minimum element spacing constraint can be set to other values.

Table 4 Positions of elliptical antenna array with 20 elements

\begin{tabular}{|c|c|c|}
\hline$N=20$ & $\varphi_{n}(\stackrel{\circ}{)}, \mathrm{n}=1,2, \cdots, 20$ & PSLL (dB) \\
\hline Uniform & $\begin{array}{l}{[0,18,36,54,72,90,108,126,144,162,180,198,216,234,} \\
252,270,288,306,324,342]\end{array}$ & -6.02 \\
\hline FA in [20] & $\begin{array}{l}{[0.77,18.00,37.25,68.33,88.66,93.51,113.53,143.98,162.00} \\
179.98,180.63,198.00,216.99,248.14,264.62,273.40,289.05 \\
321.28,342.00,358.30]\end{array}$ & -11.27 \\
\hline DE & $\begin{array}{l}{[3.32,13.98,49.01,81.64,88.67,94.30,104.15,137.01,148.71} \\
171.84,179.18,185.42,191.76,216.15,250.36,266.38,275.73 \\
292.65,346.32,353.26]\end{array}$ & -11.93 \\
\hline IWO & $\begin{array}{l}{[2.58,9.39,44.11,88.75,94.43,106.79,144.37,170.24,178.09} \\
184.77,191.06,220.37,251.87,266.42,271.87,279.86,290.64 \\
318.94,347.64,356.12]\end{array}$ & -11.96 \\
\hline DIWO & $\begin{array}{l}{[3.01,9.42,34.50,46.23,69.83,83.99,95.60,108.53,135.35} \\
171.63,178.22,185.78,194.05,249.85,257.46,265.14,273.58 \\
328.64,349.70,356.48]\end{array}$ & -12.21 \\
\hline
\end{tabular}




\section{Abbreviations}

BBO: Biogeography-based optimization; DE: Differential evolution; DIWO: Differential invasive weed optimization; HDLPSO: Hierarchical Dynamic Local Neighborhood Based PSO; IWO: Invasive weed optimization; PSLLs: Peak side lobe levels; PSO: Particle swarm optimization

\section{Acknowledgements}

Not applicable.

\section{Authors' contributions}

HG conceived the idea. HG and RJ complete the algorithm. HG, MD, and LZ wrote this paper. XZ contributed to the reviewing of the manuscript. All authors read and approved the final manuscript.

\section{Funding}

This work was supported by the Natural Science Foundation of Shaanxi Province Department of Education (17JK0319) and Doctoral Scientific Research Foundation of Xi'an Polytechnic University (107020277) and Shaanxi Provincial Science and Technology Department (2018JQ4016).

\section{Availability of data and materials}

Not applicable.

\section{Competing interests}

The authors declare that they have no competing interests.

Received: 22 March 2019 Accepted: 10 October 2019

Published online: 21 November 2019

\section{References}

1. K.C. Hwang, A modified Sierpinski fractal antenna for multiband application. IEEE Antennas Wireless Propagation Lett. 6, 357-360 (2007)

2. C. Puente-Baliarda, J. Romeu, R. Pous, A. Cardama, On the behavior of the Sierpinski multiband fractal antenna. IEEE Trans. Antennas Propagation 46(4), 517-524 (1998)

3. J. Anguera, C. Borja, C. Puentea, Microstrip fractal-shaped antennas: a review. Paper presented at the 2nd European conference on antennas and propagation. (Edinburgh, 2007), pp. 11-16

4. E. Guariglia, Harmonic Sierpinski gasket and applications. Entropy 20(9), 714 (2018)

5. E. Guariglia, Entropy and fractal antennas. Entropy 18(3), 84 (2016)

6. R.L. Haupt, Thinned arrays using genetic algorithms. IEEE Trans. Antennas Propagation 42(7), 993-999 (1994)

7. Q.T. Óscar, R.I. Eva, Ant colony optimization in thinned array synthesis with minimum sidelobe level. IEEE Antennas Wireless Propagation Lett. 5, 349-352 (2006)

8. N. Pathak, G.K. Mahanti, S.K. Singh, et al., Synthesis of thinned planar circular array antennas using modified particle swarm optimization. Prog. Electromagnetics Res. Lett. 12, 87-97 (2009)

9. L.H. Abderrahmane, B. Boussouar, New optimization algorithm for planar antenna array synthesis. Int. J. Electron. Commun. 66, 752-757 (2012)

10. W.P.M.N. Keizer, Large planar array thinning using iterative FFT techniques. IEEE Trans. Antennas Propagation 57(10), 3359-3362 (2009)

11. K.S. Chen, Z.S. He, C.L. Han, A modified real GA for the sparse linear array synthesis with multiple constraints. IEEE Trans. Antennas Propagation 54(7), 2169-2173 (2006)

12. H. Chen, Q. Wan, Non-uniform array pattern synthesis using reweighted I1-norm minimization. Int. J. Electron. Commun. 67, 795-798 (2013)

13. O.M. Bucci, T. Isernia, A.F. Morabito, An effective deterministic procedure for the synthesis of shaped beams by means of uniform-amplitude linear sparse arrays. IEEE Trans. Antennas Propagation 61(1), 169-175 (2013)

14. B. Preetham Kumar, G.R. Branner, Design of unequally spaced arrays for performance improvement. IEEE Trans. Antennas Propagation 47(3), 511-523 (1999)

15. M.A. Panduro, A.L. Mendez, R. Dominguez, et al., Design of non-uniform circular antenna arrays for side lobe reduction using the method of genetic algorithms. Int. J. Electron. Commun. 60, 713-717 (2006)

16. P. Ghosh, J. Banerjee, S. Das, et al., Design of non-uniform circular antenna arrays-an evolutionary algorithm based approach. Prog. Electromagnetics Res. B 43, 333-354 (2012)
17. G.G. Roy, S. Das, P. Chakraborty, et al., Design of non-uniform circular antenna arrays using a modified invasive weed optimization algorithm. IEEE Trans. Antennas Propagation 59(1), 110-118 (2011)

18. U. Singh, T.S. Kamal, Design of non-uniform circular antenna arrays using biogeography-based optimization. IET Microwaves Antennas Propagation 5(11), 1365-1370 (2010)

19. A. Neyestanak, M. Ghiamy, M. Moghaddasi M, et al., An investigation of hybrid elliptical antenna arrays. IET Microwaves Antennas Propagation 2(1), 28-34 (2008)

20. A. Sharaqa, N. Dib, Position-only side lobe reduction of a uniformly excited elliptical antenna array using evolutionary algorithms. IET Microwaves Antennas Propagation 7(6), 452-457 (2013)

21. S.K. Mahto, A. Choubey, A novel hybrid IWO/WDO algorithm for nulling pattern synthesis of uniformly spaced linear and non-uniform circular array antenna. Int. J. Electron. Commun. 70, 750-756 (2016)

22. S. Karimkashi, A.A. Kishk, Invasive weed optimization and its features in electromagnetics. IEEE Trans. Antennas Propagation 58(4), 1269-1278 (2010)

23. A.R. Mehrabian, C. Lucas, A novel numerical optimization algorithm inspired from weed colonization. Ecol. Inform. 1, 355-366 (2006)

24. R. Stron, K. Price, Differential evolution-a simple and efficient heuristic for a global optimization over continuous spaces. J. Glob. Optim. 11(4), 341-359 (1997)

\section{Publisher's Note}

Springer Nature remains neutral with regard to jurisdictional claims in published maps and institutional affiliations.

\section{Submit your manuscript to a SpringerOpen ${ }^{\circ}$ journal and benefit from:}

- Convenient online submission

- Rigorous peer review

- Open access: articles freely available online

High visibility within the field

- Retaining the copyright to your article

Submit your next manuscript at $\boldsymbol{\nabla}$ springeropen.com 